\title{
July 2014 Imaging Case of the Month
}

\author{
Michael B. Gotway, MD \\ Prasad M. Panse, MD \\ Department of Radiology \\ Mayo Clinic Arizona \\ Scottsdale, AZ
}

Clinical History: A 71-year-old Caucasian man presented for venous thromboembolism prophylaxis for a scheduled total right knee arthroplasty. His prior medical history was remarkable for anemia requiring transfusion of 4 units of blood 3 years prior to presentation, hypertension, prostatic hypertrophy, seasonal allergies, and glucose intolerance.

Frontal and lateral chest radiography (Figure 1) was performed.
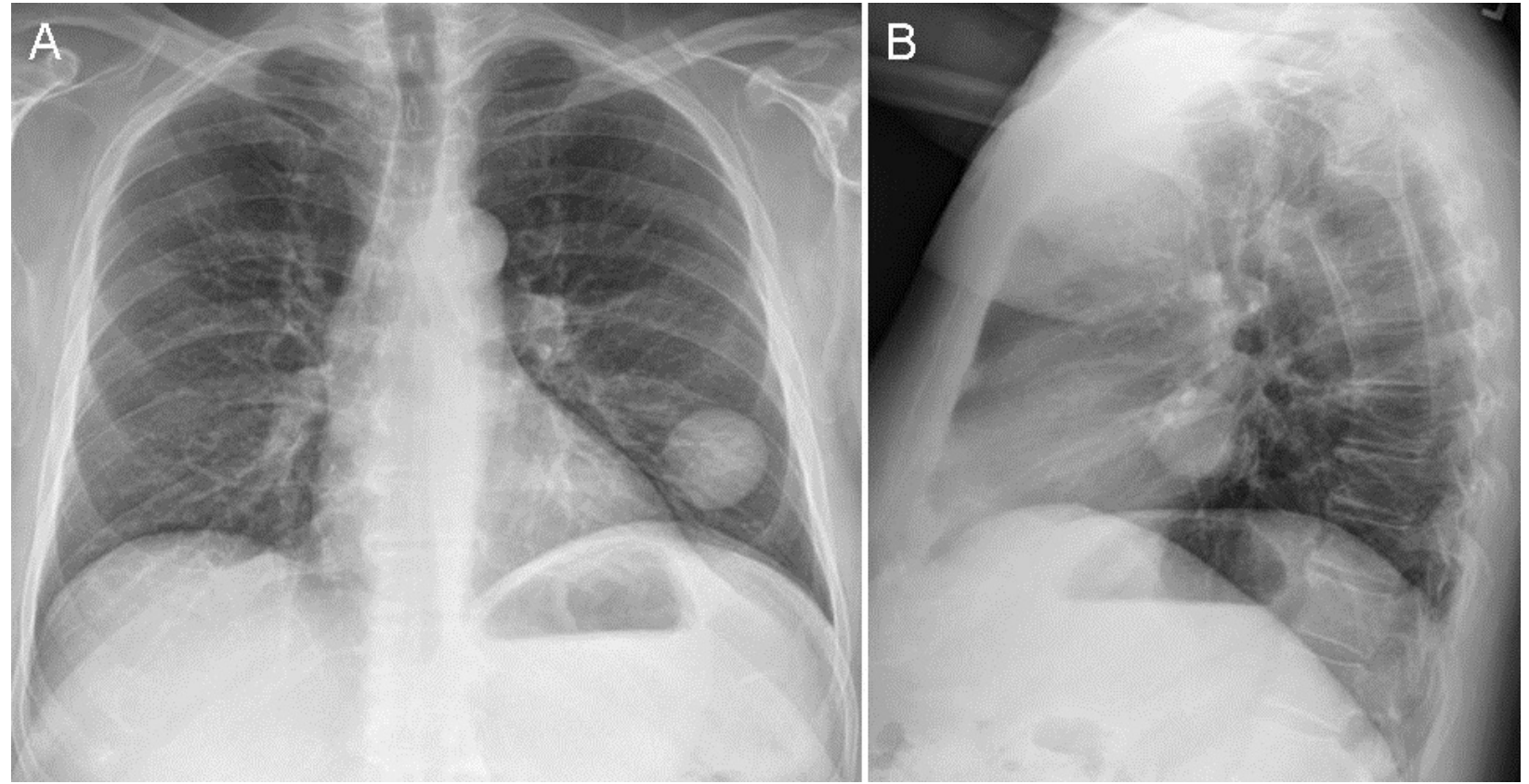

Figure 1. Frontal (Panel A) and lateral (Panel B) chest radiography.

Which of the following statements regarding the chest radiograph is most accurate?

1. The chest radiograph shows a circumscribed left lung mass

2. The chest radiograph shows a mediastinal mass

3. The chest radiograph shows bilateral symmetric linear and reticular opacities

4. The chest radiograph shows multifocal, bilateral consolidation

5. The chest radiograph shows numerous small nodules 


\section{Correct!}

\section{The chest radiograph shows a circumscribed left lung mass}

The frontal chest radiograph shows a circumscribed lingular mass. Small nodules are not present, nor is there evidence of a mediastinal abnormality or interstitial lung disease. No consolidation is present.

Which of the following represents the next, most appropriate step for the assessment of the findings at chest radiography?

1. ${ }^{18}$ FDG-PET scan

2. Bilateral, frontal shallow $\left(5^{\circ}\right)$ oblique images to distinguish true lung infiltration from artifact or a chest wall lesion

3. Fluoroscopic-guided percutaneous transthoracic needle biopsy

4. Thoracic CT scan

5. Thoracic MRI 


\section{Correct! \\ 4. Thoracic CT scan}

Thoracic MRI can be useful for the evaluation of mediastinal abnormalities, disorders of the chest wall, and occasionally even pleural space conditions, but plays a limited role for the assessment of focal lung parenchymal abnormalities. Thoracic MRI could potentially characterize the lingular lesion, but generally thoracic CT is more commonly employed for lung parenchymal lesions. The chest radiograph clearly show a lung lesion is present [there is no chance this lesion is artifactual]; therefore, bilateral frontal shallow oblique images are would not provide additional information for this patient. ${ }^{18}$ FDG-PET scanning could be used to evaluate the lingular lesion in this patient, but morphological characterization of the lesion with thoracic CT will be required as well, regardless of the ${ }^{18}$ FDG-PET scan results. If the ${ }^{18}$ FDG-PET scan does not show elevated tracer uptake, a substantial imaging abnormality remains and requires assessment; if the ${ }^{18}$ FDG-PET shows elevated tracer utilization, morphologic characterization is typically still required as elevated glucose utilization at ${ }^{18}$ FDG-PET, while often suggestive of malignancy, is not specific for malignancy, and infections and non-infectious inflammatory conditions, and even occasionally some benign lung neoplasms, may results in increased tracer utilization at ${ }^{18}$ FDG-PET. Finally, while percutaneous transthoracic needle biopsy can be performed accurately using fluoroscopic guidance, typically lesions undergoing fluoroscopic-guided needle biopsy are first characterized using thoracic CT to both confirm that the lesion undergoing biopsy is truly indeterminate (i.e., no fat or calcium is present, which would allow the diagnosis of a benign lesion and mandate conservative management), and that a lesion potentially inappropriate for transthoracic biopsy, such as a fluid-filled cyst or arteriovenous malformation, is not the cause of the lesion. Clinical course: Although prior outside imaging was not available for direct review, prior chest radiographic reports indicated that the lesion has been present for at least 10 years, although it may have enlarged compared with a chest radiograph performed three-to-four years earlier.

Additional Clinical Course: The patient subsequently underwent unenhanced thoracic CT (Figure 2). 


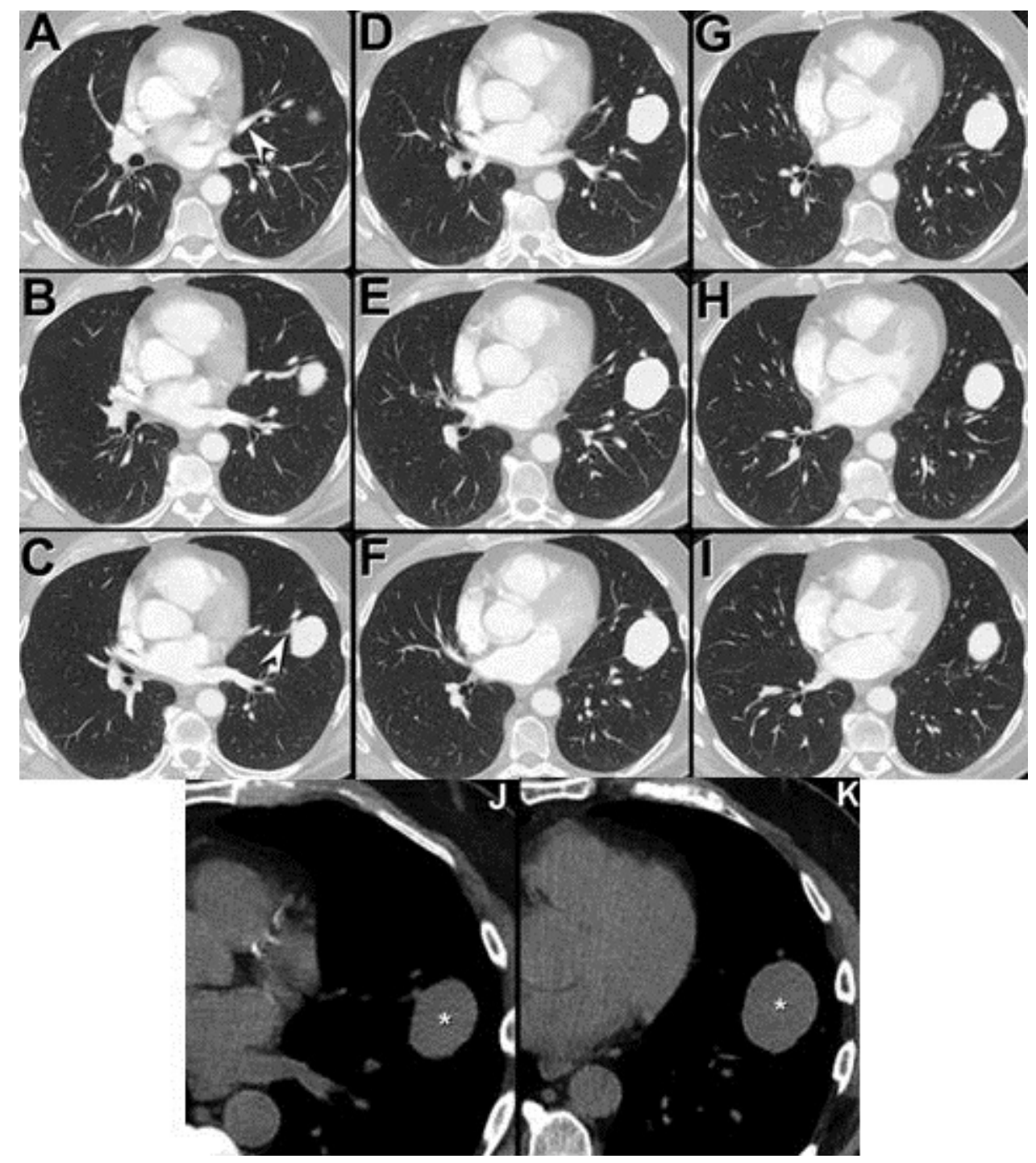

Figure 2. Axial unenhanced thoracic CT displayed in lung (A-I) and soft tissue windows $(\mathrm{J}$ and $\mathrm{K})$ shows the circumscribed lingular lesion $\left(^{*}\right)$.

Which of the following statements regarding this imaging study is most accurate?

1. The thoracic CT shows a lingular nodule associated with adjacent tubular structures representing dilated, impacted bronchi

2. The thoracic CT shows a lingular nodule associated with adjacent tubular structures representing an enlarged artery and vein

3. The thoracic CT shows a lingular nodule associated with fat, typical of hamartoma.

4. The thoracic CT shows a poorly defined lingular mass suspicious for primary bronchogenic malignancy

5. The thoracic CT shows an indeterminate, non-specific lingular nodule 


\section{Correct! \\ 2. The thoracic CT shows a lingular nodule associated with adjacent tubular structures representing an enlarged artery and vein}

The unenhanced thoracic CT shows that the lingular lesion is circumscribed, not poorly defined; the morphology, while not absolutely exclusive of malignancy, is not overtly suggestive of that diagnosis. However, tubular structures are closely associated with the lingular lesion. Typically such tubular structures represent either dilated, impacted bronchi or enlarged vasculature. When dilated bronchi are responsible for such tubular opacities, often other dilated, non-impacted bronchi are evident elsewhere, and air trapping, manifest as inhomogeneous lung opacity on inspiratory CT scanning, is also evident- however, neither finding is present in this case. Therefore, the tubular opacities in this patient are due to enlarged adjacent vasculature. There is no evidence of either fat or calcium on the soft tissue windows (Figure $2 \mathrm{~K}$ and $\mathrm{J}$ of this study).

Which of the following statements regarding the findings of this CT examination is correct?

1. The thoracic CT findings are diagnostic of arteriovenous malformation

2. The thoracic CT findings are suggestive of arteriovenous malformation, but establishing that diagnosis requires a contrast-enhanced examination

3. The thoracic CT findings are suggestive of arteriovenous malformation, but establishing that diagnosis requires a catheter pulmonary angiogram

4. The thoracic CT findings are suggestive of arteriovenous malformation, but establishing that diagnosis requires a positive echocardiographic bubble study

5. The thoracic CT findings are suggestive of arteriovenous malformation, but establishing that diagnosis requires systemic shunting to be visualized on a ${ }^{99 \mathrm{~m}} \mathrm{Tc}$-macroaggregated albumin ventilation-perfusion scintigraphy study 


\section{Correct!}

\section{The thoracic $\mathrm{CT}$ findings are diagnostic of arteriovenous malformation}

The thoracic CT findings are diagnostic of arteriovenous malformation. Establishing this diagnosis at thoracic CT requires demonstrating a circumscribed, often peripheral, nodule or mass associated with one or more enlarged supplying arteries and one or more draining veins; the diagnosis of an arteriovenous malformation is a morphological one. While demonstrating enhancement of the nodule, or nidus, provides reinforcing evidence of the presence of an arteriovenous malformation, it is not required for diagnosis. In some cases, the nidus may be thrombosed, in which case enhancement of the nidus may not be apparent, but the diagnosis may nevertheless be established through appreciation of the characteristic morphological features of arteriovenous malformation. Catheter pulmonary angiography can also establish the diagnosis of arteriovenous malformation, but is not required for this purpose- the typical CT manifestations of arteriovenous malformation are sufficient in this regard. Both a positive echocardiographic bubble study and systemic shunting to be visualized on a ${ }^{99 m}$ Tc-macroaggregated albumin ventilation-perfusion scintigraphy study are evidence of right-to-left shunting, but neither are specific for the diagnosis of arteriovenous malformation).

Further clinical course: The patient underwent contrast-enhanced thoracic CT (Figure 3) as well.

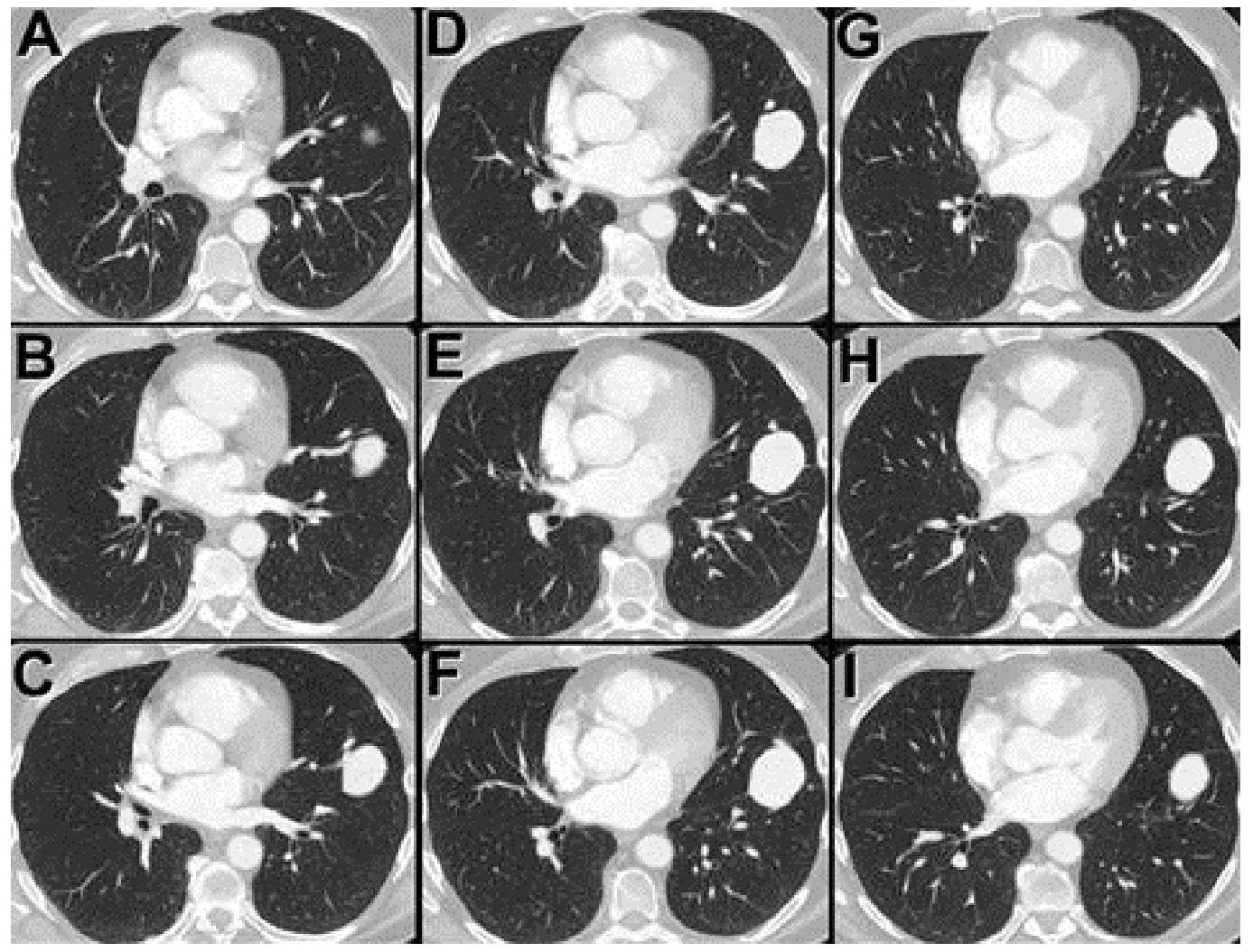

Figure 3. Axial enhanced thoracic CT scan displayed in lung windows. 
Which of the following represents the next most appropriate step for the treatment of this patient?

1. Assessment of shunt via breathing $100 \%$ oxygen with therapy only if significant shunt is demonstrated

2. Catheter pulmonary angiography with coil or detachable balloon embolization of the arteriovenous malformation

3. Percutaneous transthoracic CT-guided placement of coils or plugs within the lingular arteriovenous malformation

4. Thoracotomy for resection of the lingular arteriovenous malformation

5. Video-assisted thoracoscopic resection of the lingular arteriovenous malformation 


\section{Correct! \\ 2. Catheter pulmonary angiography with coil or detachable balloon embolization of the arteriovenous malformation}

Catheter pulmonary angiography with coil or detachable balloon embolization of the arteriovenous malformation (typically coils or Amplatzer vascular plugs) is the therapy of choice for arteriovenous malformation. Surgical resection can be considered in selected cases not amenable to catheter pulmonary arterial embolization or for patients with arteriovenous communications that persist despite maximum embolotherapy, but is exceptional. Percutaneous placement of coils within the arteriovenous malformation is not the favored method for occlusion of the nidus; rather, endovascular therapy is the preferred route. While shunt assessment via breathing 100\% oxygen is useful for establishing the physiological significance of an arteriovenous malformation, possibly as part of a screening regimen for at-risk patients (such as those with hereditary hemorrhagic telangiectasia), and for following treated patients to assess for potential recurrence, if it not required to determine the need for therapy for a lesion of this size. For such a large lesion, shunt is almost certainly present and significant; moreover, the large size of the lesion and associated vasculature place the patient at risk for systemic embolization, and therefore therapy is required to reduce the possibility of such, particularly stroke.

The patient underwent catheter pulmonary angiography (Figure 4) and coil embolization of the lingular arteriovenous malformation.

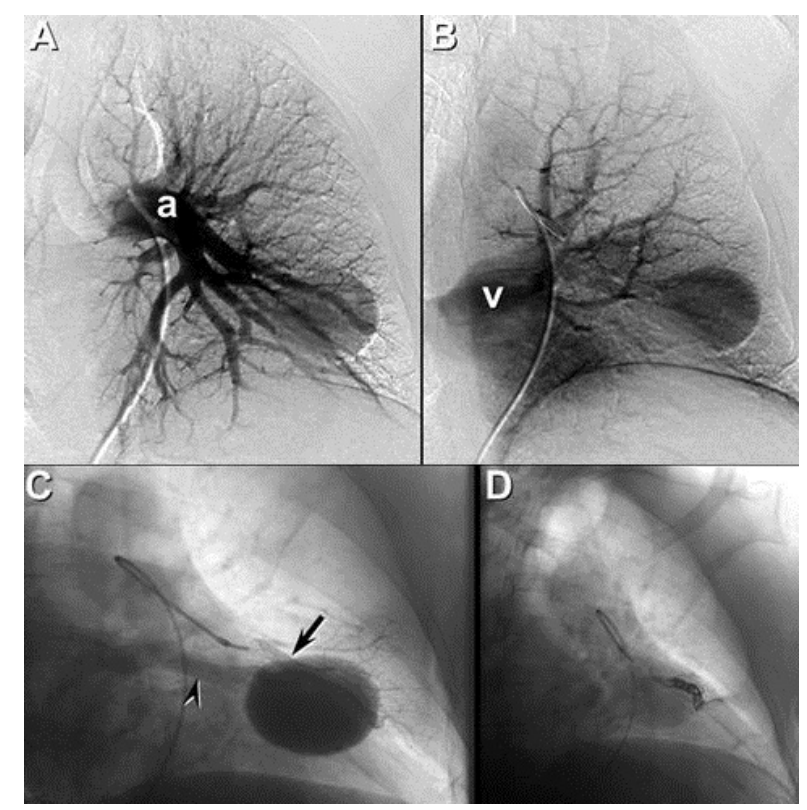

Figure 4. Catheter pulmonary angiogram in the arterial (A) and venous (B) phases just prior to placement of coils for embolization of the arteriovenous malformation shows the arterial (a) supply and central venous drainage (v). (C) Selective catheterization of the feeding artery (arrow) shows the lesion and the draining vein (arrowhead). (D) Occlusion of the feeding artery by coil embolization. 
He recovered from the procedure without difficulty and his oximetry was normal. The post-embolotherapy thoracic CT (Figure 5) showed only minimal perfusion within the large lingular vascular nidus.

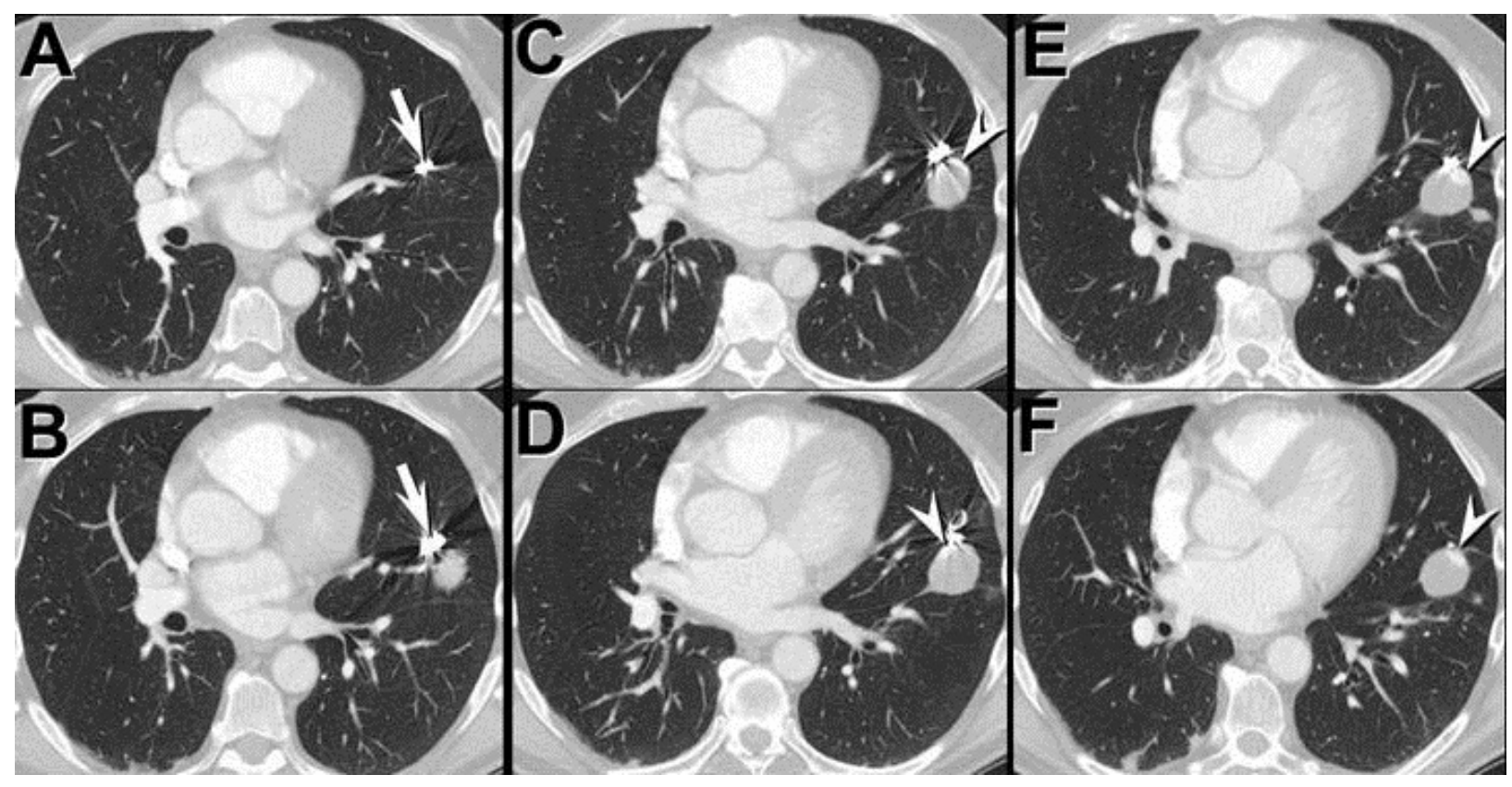

Figure 5. Axial enhanced thoracic CT displayed in lung windows, performed 6 months following coil embolotherapy, shows minimal enhancement in the anterior portion of the arteriovenous malformation (arrowheads). Coil occlusion of the feeding artery (arrows) is visible.

Diagnosis: Pulmonary arteriovenous malformation, treated with catheter pulmonary angiography embolotherapy.

\section{References}

1. Nakayama M, Nawa T, Chonan T, Endo K, Morikawa S, Bando M, Wada Y, Shioya T, Sugiyama Y, Fukai S. Prevalence of pulmonary arteriovenous malformations as estimated by low-dose thoracic CT screening. Intern Med. 2012; 51:1 677-681. [PubMed]

2. Cottin V, Plauchu H, Bayle JY, et. al. Pulmonary arteriovenous malformations in patients with hereditary hemorrhagic telangiectasia. Am J Respir Crit Care Med. 2004; 169:994-1000. [CrossRef] [PubMed]

3. Faughnan M, Palda V, Garcia-Tsao G, et al. International Guidelines for the Diagnosis and Management of Hereditary Hemorrhagic Telangiectasia. J Med Genet. 2011;48; 73-87. [CrossRef] [PubMed]

4. Dutton JAE, Jackson JE, Hughes JMB, et. al. Pulmonary arteriovenous malformations: Results of treatment with coil embolization in 53 patients. Am J Roentgenol AJR. 1995; 165:1119-1125. [CrossRef] [PubMed] 
5. Remy J, Remy-Jardin M, Wattinne L, Deffontaines C. Pulmonary arteriovenous malformations: Evaluation with CT of the chest before and after treatment.

Radiology. 1992; 182:809-816. [PubMed] 